\title{
The Prevalence and Incidence of Insomnia in Korea during 2005 to 2013
}

\author{
Seockhoon Chung ${ }^{1 *}$, Seung Woo $\mathrm{Cho}^{2 *}$, Min-Woo $\mathrm{Jo}^{3}$, Soyoung Youn ${ }^{1}$, Jiho Lee ${ }^{2}$, and Chang Sun Sim ${ }^{2}$ \\ ${ }^{1}$ Department of Psychiatry, Asan Medical Center, University of Ulsan College of Medicine, Seoul, Republic of Korea \\ ${ }^{2}$ Department of Occupational and Environmental Medicine, Ulsan University Hospital, University of Ulsan College of Medicine, \\ Ulsan, Republic of Korea \\ ${ }^{3}$ Department of Preventive Medicine, University of Ulsan College of Medicine, Seoul, Republic of Korea
}

Objective The aim of this study was to estimate the progress of insomnia prevalence and incidence over the past several years. Also, this study compared survival rates between individuals with and without insomnia.

Methods The National Health Insurance Service-National Sample Cohort (NHIS-NSC) from 2002-2013 was used for this study. Prevalent cases of insomnia were defined using ICD-10 codes F51.0 or G47.0, or a prescription of sedatives. Cox's proportional hazard analysis was conducted to compare survival rates between insomnia patients and people without insomnia.

Results In 2013, there were 46,167 (5.78\%) insomnia patients over 20 years old in this cohort. Insomnia was more common among women and the elderly. Annual incidence over the past several years remained steady but the prevalence increased. The survival of insomnia patients was lower than that of people without insomnia, and the hazard ratio for overall mortality was $1.702(\mathrm{p}<0.001)$.

Conclusion This large-scale population-based cohort study provided current epidemiologic indicators of insomnia in the Korean general population.

Psychiatry Investig 2020;17(6):533-540

Key Words Insomnia, Incidence, Prevalence, Mortality.

\section{INTRODUCTION}

Insomnia is a common symptom frequently observed in the clinic. The annual prevalence of insomnia symptoms and insomnia disorder has been reported to be between $35-50 \%{ }^{1}$ and $10-22 \%^{2,3}$ in the United States. In South Korea, the prevalence of insomnia symptoms and diagnosis (DSM-IV) were reported to be $17-23 \%$ and $5 \%$, respectively. ${ }^{4,5} \mathrm{~A}$ female predisposition to insomnia has consistently been reported, and the prevalence has increased over time amongst the elderly popu-

\footnotetext{
Received: August 20, 2019 Revised: February 3, 2020 Accepted: March 4, 2020

$\triangle$ Correspondence: Chang Sun Sim, MD, PhD

Department of Occupational and Environmental Medicine, Ulsan University Hospital, University of Ulsan College of Medicine, 877 Bangeojinsunhwandoro, Dong-gu, Ulsan 44033, Republic of Korea

Tel: +82-52-250-8933, Fax: +82-52-250-7289, E-mail: zzz0202@naver.com

$\triangle$ Correspondence: Seockhoon Chung, MD, PhD

Department of Psychiatry, Asan Medical Center, University of Ulsan College of Medicine, 86 Olympic-ro 43-gil, Songpa-gu, Seoul 05505, Republic of Korea Tel: +82-2-3010-3411, Fax: +82-2-485-8381, E-mail: schung@amc.seoul.kr

*These authors contributed equally to this work.

(a) This is an Open Access article distributed under the terms of the Creative Commons Attribution Non-Commercial License (https://creativecommons.org/licenses/by$\mathrm{nc} / 4.0$ ) which permits unrestricted non-commercial use, distribution, and reproduction in any medium, provided the original work is properly cited.
}

lation. ${ }^{6}$ Also, the prevalence has increased among inpatients compared to outpatients. ${ }^{7,8}$ People suffering from insomnia symptoms report worsened quality of life in various domains ${ }^{9,10}$ and tend to develop other psychiatric disorders. ${ }^{11}$ Recent studies have consistently showed correlations between insomnia and numerous medical problems, such as obesity, diabetes, cardiovascular disease, and even cancers. ${ }^{12-14}$

In addition to these adverse health outcomes of insomnia, potential over-usage of sleeping pills without proper instruction, ${ }^{15}$ may raise a public health concern. Furthermore, insomnia and hypnotics usage, despite the controversy, may increase the mortality rate. ${ }^{16,17}$ Therefore, it is important to explore changes in the prevalence and incidence of insomnia over the past several years in order to increase the knowledge base concerning sleep health.

In a previous study done in Sweden, the prevalence of insomnia among men in the general population was reported as increasing from $10.3 \%$ in 1984 to $12.8 \%$ in $1994 .{ }^{18}$ In Norway, the prevalence of insomnia as defined by the DSM-IV diagnostic criteria was reported to have increased from $11.9 \%$ in 1999 to $15.5 \%$ in $2009 .{ }^{19}$ In the United States, the prevalence increased from $17.5 \%$ in 2002 to $19.2 \%$ in $2012 .{ }^{20}$ In Taiwan, 
the prevalence of insomnia increased from $2.5 \%$ in 2002 to $4.2 \%$ in $2009 .^{21}$ The definition criteria of insomnia varied from study to study; however, taken together, the findings of these studies indicate that during the past several years, insomnia prevalence has been increasing worldwide.

When we use national health insurance data, we can observe changes in the prevalence and incidence of insomnia over several time periods. Despite this advantage, it is not necessarily easy to apply the diagnostic criteria for insomnia to this data. In South Korea, when physicians decide to prescribe hypnotics, the diagnosis is coded according to the International Classification of Disease, Tenth Revision (ICD-10). However, it is unclear from this data whether the ICD-10 codes for insomnia (F51.0 and G47.0) are appropriately applied or not, and sometimes coding is omitted. In these cases, we can estimate the pattern of insomnia prevalence or incidence by looking at the pattern or history of hypnotics prescriptions. Physicians usually prescribe sleeping pills approved by the appropriate drug regulatory agencies of each nation, such as z-class drugs or triazolam, but they sometimes prescribe hypnotic medications without regulatory approval, including benzodiazepines, hypnotic antidepressants, antihistamines, or antipsychotics. The aim of this study was to estimate the progress of insomnia prevalence and incidence over the past several years (2002-2013) from the Korean National Health Insurance claims data, and to explore whether insomnia is associated with mortality or not.

\section{METHODS}

\section{Study population}

The National Health Insurance Service-National Sample Cohort (NHIS-NSC) from 2002-2013 was used for this study. ${ }^{22}$ This cohort is a nationally representative random sample comprised of 1,025,340 individuals accounting for the all Korean population of $46,605,433$ in 2002 according to the National Health Insurance Database. Deceased individuals in the NHISNSC were replaced with additions of infants (age 0) in order to maintain the representativeness of the Korean general population. This cohort includes four databases on participants' insurance eligibility, medical treatments, medical care institutions, and general health examinations. Fifty-seven variables are available in the medical treatment database, such as participants' electronic medical treatment bills, bill details, details of diseases, and details of prescriptions. More details were described elsewhere. ${ }^{22}$ All research procedures were approved by the Ulsan University Hospital Institutional Review Board (UUH 2014-08-008-012).

\section{Definition of prevalent and incident cases of insomnia}

Participants who had insomnia between 2005 and 2013 were selected from this cohort. Prevalent cases were defined if hypnotic drugs were prescribed for more than 7 days or if ICD-10 codes F51.0 (nonorganic insomnia) and G47.0 (disorders of initiating and maintaining sleep, insomnias) were applied as either a principal or an additional diagnosis at least one time. Hypnotic drugs included doxepin, flunitrazepm, flurazepam, triazolam, zolpidem, and trazodone. Lorazepam, etiazolam, or quetiapine also are prescribed as sleeping pills for some patients. However, those medications are widely used for anxiety or delirium, too. So we exclude those medications from the hypnotics list to prevent false positive. A denominator of prevalence was the eligible people in the cohort of 2013. Incident cases were defined as prevalent cases who had no prior diagnosis or hypnotics usage for at least 3 years prior to the beginning of the case-defining period. The eligible population of the 2013 cohort was a denominator of incidence rate after the exclusion of prevalence who had no prior diagnosis for at least 3 years prior to the beginning of the case-defining period.

\section{Statistical analyses}

Annual standardized prevalence and incident rates of insomnias over 20 years old were described according to gender and age in 2013. For each prevalence and incidence, the mid-year population of 2013 was used as a denominator of standardized rate. To provide all available data, the number of annual prevalent and incident cases was used from 2002 to 2013 and from 2005 to 2013 for age and gender comparisons, respectively. Each standard error of standardized prevalence and incidence was estimated using the Keyfitz's formula. ${ }^{23}$ For survival analysis, the incident cases of 2008 were followed up from the first diagnosis until death or December 2013. The reason why incidence of 2008 was selected was to explore the latest 5 year survival on insomnia. Patients were matched 1:4 to randomly select non-case controls from the 2008 NHISNSC by age and gender. These individuals were followed up from January 2008 to their death dates or December 2013. Descriptive data were provided on groups and chi-square tests were conducted to examine differences of distributions in general characteristics. And, using these data, Kaplan-meier analysis and Cox's proportional hazard analysis were conducted. The Statistical Package for the Social Sciences (SPSS) version 21.0 (IBM Corp., Armonk, NY, USA) used for these statistical analyses.

\section{RESULTS}

In $2013,46,167$ (5.78\%) insomnia patients were included 
over 20 years old in this cohort. Among them, 43,961 had ICD-10 codes F51.0 and G47.0, and 2,206 received hypnotics prescriptions only without an ICD-10 diagnosis. The prevalence of insomnia in 2013 was $7.20 \%$ and $4.32 \%$ in females and males, respectively.

Figures 1 and 2 show the incident and prevalence rates of insomnia based on age and gender. More women than men had insomnia, and there were generally more incident and prevalent cases in the older age groups than in the lower age groups. In 2013, the highest incident and prevalence rates of insomnia based on gender and age groups are 80 years. The gender-related difference in insomnia risk attenuated after age 80 and older.

The standardized incidence rate of insomnia was slightly increased during the study period, but the increase of standardized prevalence rate was obvious for both genders. In 2005 and 2013, the incidences of insomnia were 2.92\% [95\% confidence interval (CI): $2.86-2.98 \%$ ] and $3.11 \%$ (95\% CI:

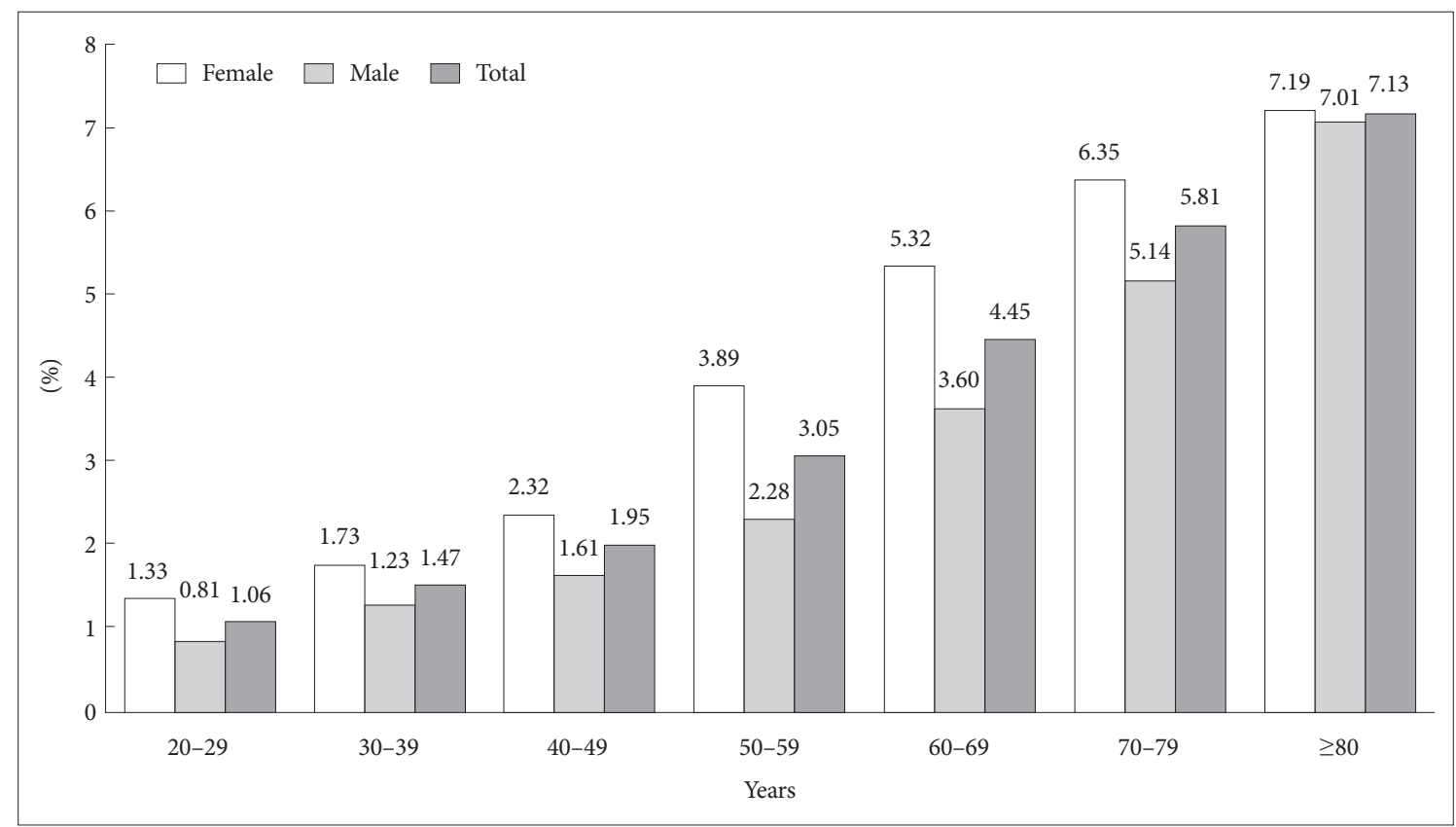

Figure 1. Incidence of insomnia on gender and age group in 2013.

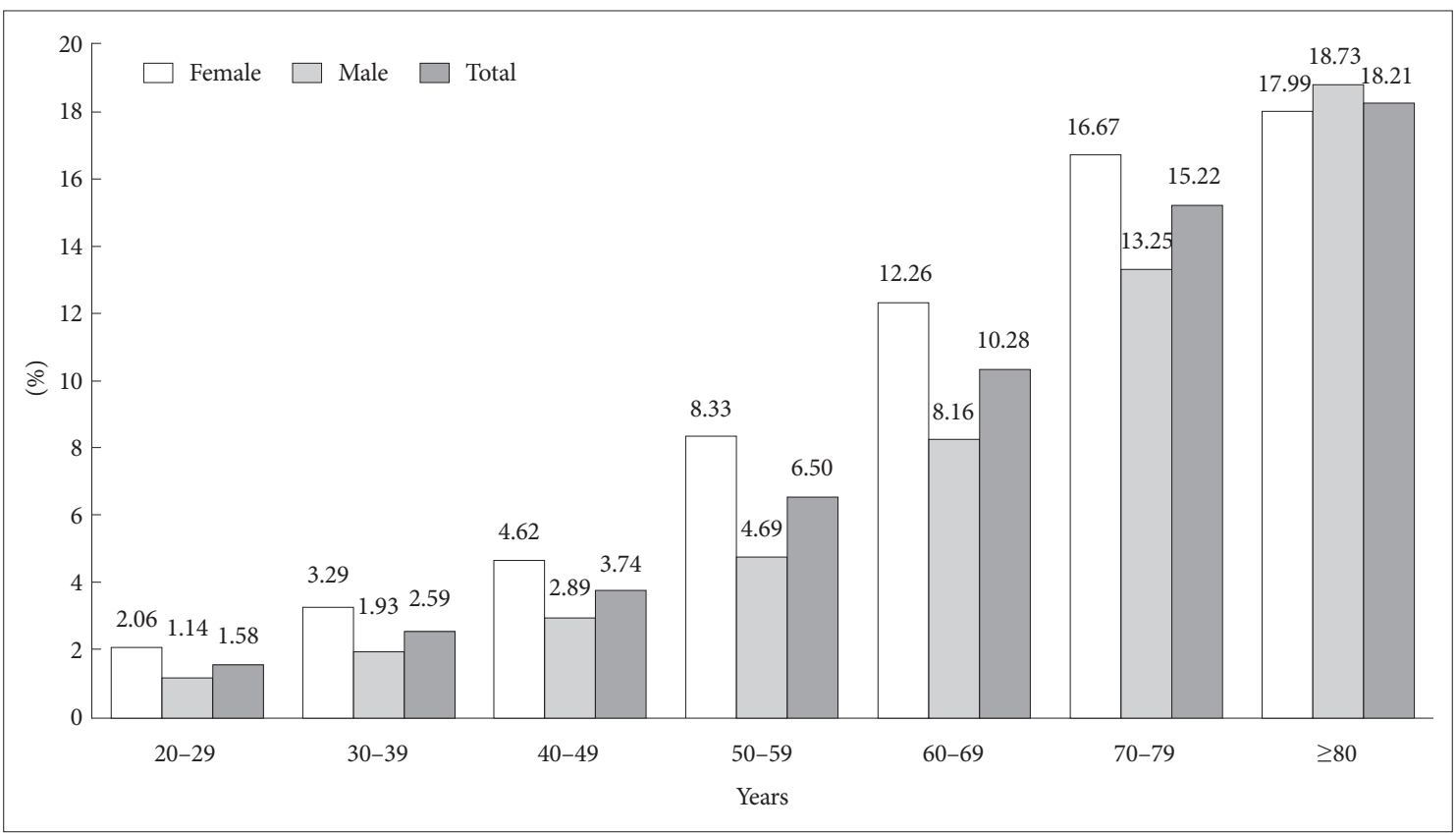

Figure 2. Prevalence of insomnia on gender and age group in 2013. 
$3.05-3.17 \%)$ in females and $1.67 \%$ (95\% CI: $1.63-1.71 \%)$ and $2.01 \%$ (95\% CI: 1.96-2.06\%) in males, respectively (Figure 3). However, the standardized prevalence more than doubled from 2002 to 2013 [3.10\% (95\% CI: 3.16-3.04\%) to $7.20 \%$ (95\% CI: 7.12-7.28\%) in females and 1.62\% (95\% CI: 1.58-1.66\%) to $4.32 \%$ (95\% CI: $4.26-4.38 \%$ ) in males] (Figure 4 ).

Table 1 shows general characteristics of insomnia group and non-case control group. There were no statistical differences on gender, age and health insurance premium. In noncase group, those living in metropolitan areas in insomnia were $1.6 \%$ more likely than those in non-case control group $(\mathrm{p}<0.001)$. All observed person years (PYs) at 60 months was 343,004 PYs in the control group and 84,341 PYs in the insomnia group, respectively. And dead cases were 2,446 (3.5\%) and 985 (5.7\%), respectively.

The results of the survival analysis are presented in Figure 5. According to the Kaplan-meier analysis, there was a statistically significant difference in survival rate on individuals of insomnia $(\mathrm{p}<0.001)$. The survival of patients with insomnia was lower than that of patients without insomnia (95.0\% and $97.3 \%$, respectively, at 60 months). Overall hazard ratio was 1.702 (95\% CI: 1.580-1.833, p<0.001) from Cox's proportional hazard analysis.

\section{DISCUSSION}

In this study, we observed that the prevalence of insomnia increased over the past several years in South Korea, and it is

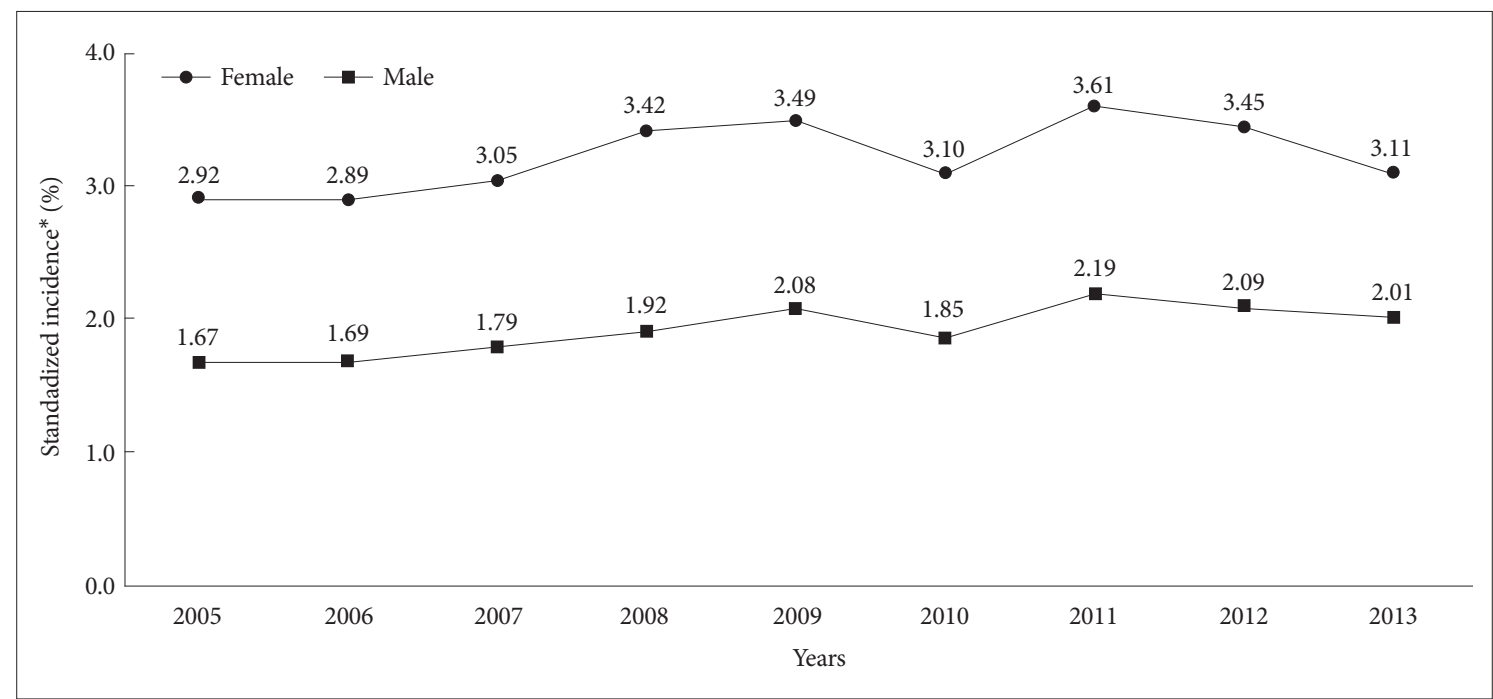

Figure 3. Standardized incidence with insomnia on gender from 2005 to 2013 . *Age and gender standardized rate using the mid-year population of 2013.

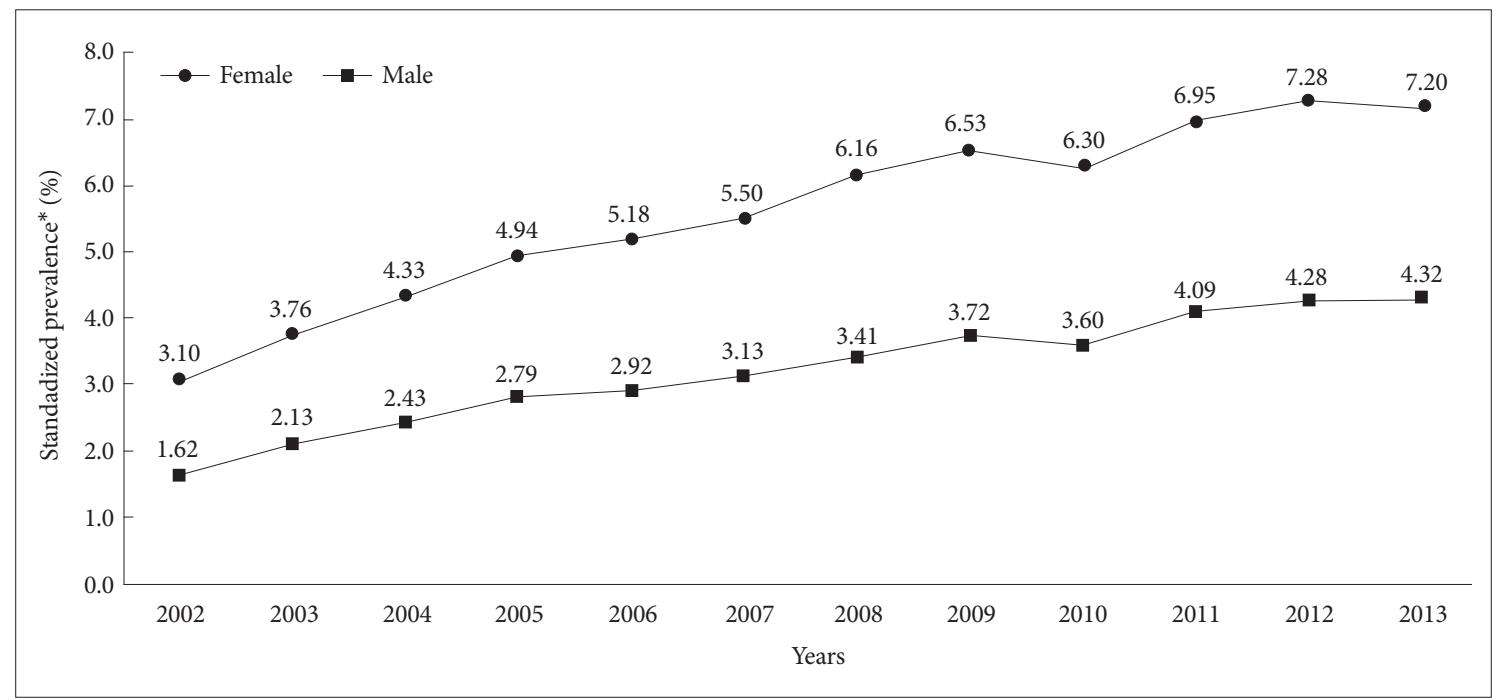

Figure 4. Standardized prevalence with insomnia on gender from 2002 to $2013 .{ }^{*}$ Age and gender standardized rate using the mid-year population of 2013 . 
Table 1. General characteristics of insomnia group and non-case control group

\begin{tabular}{|c|c|c|c|}
\hline & $\begin{array}{c}\text { Non-case } \\
\text { control, } \\
\mathrm{N}(\%)\end{array}$ & $\begin{array}{l}\text { Insomnia, } \\
\mathrm{N}(\%)\end{array}$ & $\begin{array}{c}\mathrm{p}- \\
\text { value }\end{array}$ \\
\hline Sex & & & 1.000 \\
\hline Male & $25,076(36.1)$ & $6,269(36.1)$ & \\
\hline Female & $44,388(63.9)$ & $11,097(63.9)$ & \\
\hline Age & & & 1.000 \\
\hline $20 \mathrm{~s}$ & $5,424(7.8)$ & $1,356(7.8)$ & \\
\hline $30 \mathrm{~s}$ & $9,128(13.1)$ & $2,282(13.1)$ & \\
\hline $40 \mathrm{~s}$ & $13,756(19.8)$ & $3,439(19.8)$ & \\
\hline $50 \mathrm{~s}$ & $14,268(20.5)$ & $3,567(20.5)$ & \\
\hline $60 \mathrm{~s}$ & $12,692(18.3)$ & $3,173(18.3)$ & \\
\hline $70 \mathrm{~s}$ & $10,224(14.7)$ & $2,556(14.7)$ & \\
\hline 80 and over & $3,972(5.7)$ & $993(5.7)$ & \\
\hline Region & & & $<0.001$ \\
\hline Metropolitan areas & $33,028(47.5)$ & $7,976(45.9)$ & \\
\hline City \& rural areas & $36,436(52.5)$ & $9,390(54.1)$ & \\
\hline Health insurance premium & & & 0.726 \\
\hline High & $29,542(42.5)$ & $7,360(42.4)$ & \\
\hline Low & $39,922(57.5)$ & $10,006(57.6)$ & \\
\hline Survival at 60 months & & & $<0.001$ \\
\hline Alive & $67,018(96.5)$ & $16,381(94.3)$ & \\
\hline Death & $2,446(3.5)$ & $985(5.7)$ & \\
\hline $\begin{array}{l}\text { Observed person years } \\
\text { at } 60 \text { months }\end{array}$ & 343,004 & 84,341 & - \\
\hline
\end{tabular}

comparable to the previous study which was done in Taiwan. ${ }^{21}$

The prevalence of insomnia in 2013 was $7.20 \%$ and $4.32 \%$ in females and males, respectively.

More women than men had insomnia, and there were generally more incident and prevalent cases in the older age groups than in the lower age groups. Although the incidences of insomnia were $2.92 \%$ (95\% CI: 2.86-2.98\%) in female and 1.67\% (95\% CI: $1.63-1.71 \%$ ) in males in 2005, and 3.11\% (95\% CI: $3.05-3.17 \%$ ) in female and $2.01 \%$ (95\% CI: $1.96-2.06 \%$ ) in males in 2013, respectively, the standardized prevalence more than doubled from 2002 to 2013 [3.10\% (95\% CI: 3.16-3.04\%) to $7.20 \%$ (95\% CI: 7.12-7.28\%) in females and $1.62 \%(95 \%$ CI: $1.58-1.66 \%$ ) to $4.32 \%$ (95\% CI: $4.26-4.38 \%$ )]. The results of the survival analysis are presented that the survival of patients with insomnia was lower than that of patients without insomnia.

The prevalence of insomnia has been obviously increasing over time in this study. In modern society, people's increased psychological stress may influence the increased prevalence of insomnia. ${ }^{24,25}$ Heavy workloads, alternative work schedules,

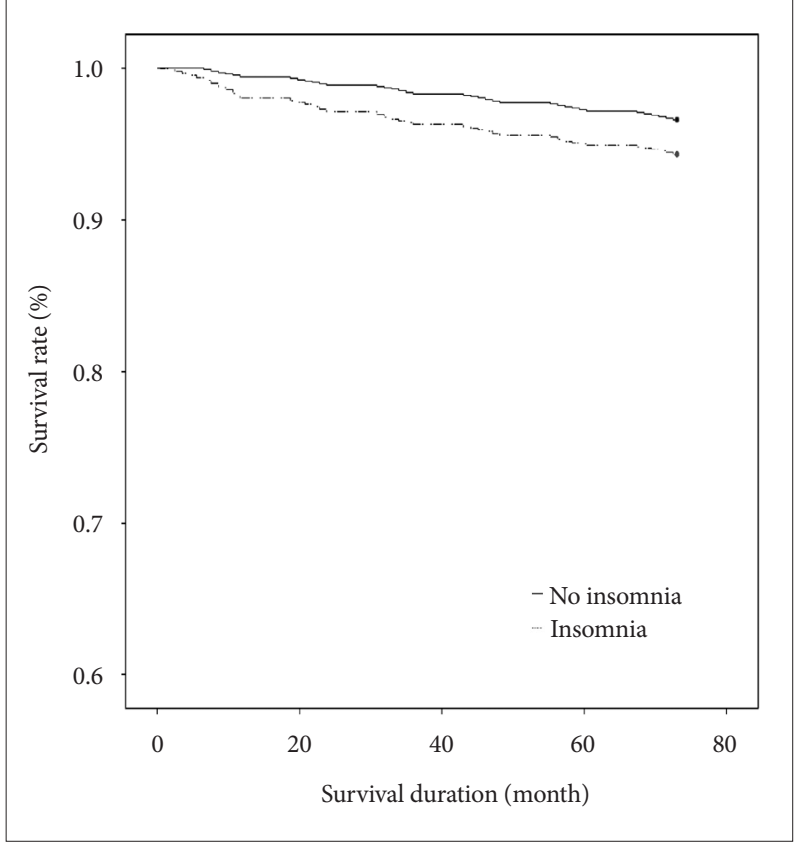

Figure 5. Survival rates between individuals with and without insomnia.

and high-caffeine drink consumption also can affect insomnia. ${ }^{21,26-28}$ Insomnia symptom may be also related to certain psychiatric or medical conditions. For instance, insomnia is a common, subjective sleep complaint of patients with depressive or anxiety disorders. ${ }^{29,30}$ Primary sleep disorders such as obstructive sleep apnea or periodic limb movement disorder can also induce insomnia. Thus, we also may explore whether the increasing prevalence and incidence rates of hypnotic drug prescription in the past 10 years came from insomnia itself or other sleep disorders.

In South Korea, changes in sleep disorder prevalence from $1.11 \%$ (men) and $1.97 \%$ (women) in 2004 to $1.41 \%$ (men) and $2.14 \%$ (women) in 2009, based on data from national health insurance service, have been reported. ${ }^{31}$ First, the number of patients treated for depression, a major psychiatric disease that can induce insomnia, increased dramatically from about 516,000 in 2009 to about 603,000 in 2015 according to claims data from the National Health Insurance Service of Korea. Second, among primary sleep disorder which can influence insomnia symptoms, subjects who were treated for restless legs syndrome (RLS), another sleep disorder which may influence insomnia symptoms, increased among all sleep disorders from $0.13 \%$ in 2004 to $1.63 \%$ in $2009,{ }^{13}$ although this increase could be explained by people becoming more educated about RLS and thus increasing visits to the hospital. Sleep apnea is usually influenced by obesity and aging. The Korea National Health and Nutrition Examination Survey (KNHANES) reported that the prevalence of obesity, defined as body mass index $\geq 25 \mathrm{~kg} / \mathrm{m}^{2}$, increased from $26.9 \%$ in 1998 to $32.0 \%$ in 
2011..$^{32}$ Also, an increasingly aged society in Korea may raise the prevalence of OSAS, because airway collapsibility, one of the major mechanisms of OSAS, increases with age. ${ }^{33,34}$

In this study, the prevalence increased according to age, and female predominance was observed. Gender ratio in the prevalence of insomnia in this study was very similar to Taiwan study ${ }^{21}$ at which there was increase in female preponderance at the elderly, in accordance with a previous meta-analysis at which elderly has more female preponderance than young adult. Psychological problems, comorbid medical conditions, or decreased physical activity which can influence their insomnia symptoms. And also, women are more vulnerable to psychological stresses, and Additionally, greater bodily vigilance and awareness is found among women, and a socially sanctioned culture exists that encourages women to more readily express emotional distress and somatic symptoms. ${ }^{24}$

It has been repeatedly reported that insomnia or hypnotics usage is associated with an increased risk of mortality, ${ }^{35,36} \mathrm{de}$ spite the controversy. ${ }^{37,38}$ In this study, we observed a statistically significant difference in survival rates between insomnia patients and non-insomnia; however, we cannot conclude that insomnia (or hypnotics usage) itself increases the mortality risk among the general population from the results of this study. Various kinds of situations that induce insomnia or hypnotics usage should be considered. It is well known that patients with medical or neurological diseases that are related to higher mortality rates, such as cancers ${ }^{39}$ or Parkinson disease, ${ }^{40}$ suffer from insomnia or sleep disturbance. Patients with psychiatric disorders, such as psychotic disorders or depression, which are related with higher suicide risk, also suffer from sleep disturbances. $^{41}$

We defined insomnia as having taken hypnotic drugs for at least 7 days or as having been diagnosed according to the ICD-10 codes of insomnia (F51.0 or G47.0). In this study, 2,228 subjects were prescribed sleeping pills without any insomnia ICD-10 codes. Including individuals diagnosed with insomnia by ICD-10 codes only may have underrepresented the prevalence of insomnia, considering that physicians often prescribe sleeping pills without coding; therefore, we expanded our definition of insomnia to include individuals with a history of a prescription for sleeping pills. Hypnotic drugs included in the definition of insomnia were zolpidem, benzodiazepines, including triazolam, flunitrazepm, and flurazepam, and hypnotic antidepressants, including trazodone and doxepine.

This study has some limitations. First, we defined insomnia as using hypnotics for at least 7 days or by ICD-10 codes. It is difficult to classify insomnia precisely particularly when we use national health insurance data. For example ICD-10 code for nonorganic insomnia may be nonspecific. It is a limitation when we use national representative data. Another limitation is that we used hypnotics usage data for defining insomnia. Usually zolpidem or triazolam are prescribed for insomnia only. However, other benzodiazepines or hypnotic antidepressants can be used for anxiety, pain, or depression. In this study, we cannot exclude patients who used those medications for other reasons. Conversely, some patients who were diagnosed as having other major psychiatric diseases, such as major depressive disorder, schizophrenia, or bipolar disorder, might be prescribed other hypnotic medications, such as mirtazapine or quetiapine, for managing their major psychiatric symptoms and sleep problems at the same time. Some patients might also take over-the-counter sleeping pills, ${ }^{42}$ which are not recorded in the national health insurance system. Third, this study used health insurance claim data. Generally, the validity of claims data may be limited. For this reason, we considered hypnotics prescription in our definition of insomnia. However, many people who suffer from insomnia may not visit clinics or hospitals. Thus, the epidemiologic estimates in this study, including prevalence, incidence, and hazard ratio, could be underestimated compared to the actual levels. This work examines patterns of office visits/physician diagnosis of insomnia, rather than the true population rates. The incident cases likely do not reflect when insomnia started, but rather the first discussion/diagnosis by a physician. Finally, the increase in mortality in subjects with insomnia needs to be explained with caution. We did not show the direct causal relationship between insomnia and mortality. We did not observe the mortality among subjects with persistence insomnia, and we could not observe the effect of insomnia separated from hypnotics usage. In addition, important confoundings such as smoking were not considered because of limitation of data.

In conclusion, we have shown the annual incidence and prevalence of insomnia of recent 10 continuous years using national representative data. And also, we defined insomnia using ICD-10 codes and hypnotics usage data to observe more precisely, although we could not get the over-the-counter drug usage. We could observe increased prevalence of insomnia from $3.1 \%$ to $7.2 \%$ in females and $1.62 \%$ to $4.32 \%$ in males over recent 10 years of period. The annual incidences were $1.67-2.15 \%$ in male and $2.89-3.61 \%$ in females respectively. And also, we observed a significance difference in survival rate between individuals with insomnia and without insomnia.

\section{Acknowledgments}

We thank Ms. Vanessa Topping from the Scientific Publications Team at Asan Medical Center for her editorial assistance in preparing this manuscript.

This work was supported by the Korea Ministry of Environment (MOE) as "the Environmental Health Action Program (grant number: 201400135001)."

\section{Conflicts of Interest}

The authors have no potential conflicts of interest to disclose. 


\section{Author Contributions}

Conceptualization: Chang Sun Sim, Min-Woo Jo, Seockhoon Chung. Data curation: Seung Woo Cho, Min-Woo Jo, Jiho Lee. Formal analysis: Min-Woo Jo, Seung Woo Cho. Investigation: Seockhoon Chung, Soyoung Youn. Methodology: Seockhoon Chung, Soyoung Youn, Chang Sun Sim, Min-Woo Jo. Supervision: Seockhoon Chung, Jiho Lee. Visualization: Soyoung Youn, Min-Woo Jo, Seung Woo Cho. Writing_-original draft: Seockhoon Chung, Seung Woo Cho. Writing-review \& editing: Chang Sun Sim, Min-Woo Jo, Jiho Lee.

\section{ORCID iDs}

$\begin{array}{ll}\text { Seockhoon Chung } & \text { https://orcid.org/0000-0002-9798-3642 } \\ \text { Seung Woo Cho } & \text { https://orcid.org/0000-0001-5670-9914 } \\ \text { Min-Woo Jo } & \text { https://orcid.org/0000-0002-4574-1318 } \\ \text { Soyoung Youn } & \text { https://orcid.org/0000-0003-4983-4017 } \\ \text { Jiho Lee } & \text { https://orcid.org/0000-0001-8027-835X } \\ \text { Chang Sun Sim } & \text { https://orcid.org/0000-0003-0841-1413 }\end{array}$

\section{REFERENCES}

1. Walsh JK, Coulouvrat C, Hajak G, Lakoma MD, Petukhova M, Roth T, et al. Nighttime insomnia symptoms and perceived health in the America Insomnia Survey (AIS). Sleep 2011;34:997-1011.

2. Roth T, Coulouvrat C, Hajak G, Lakoma MD, Sampson NA, Shahly V, et al. Prevalence and perceived health associated with insomnia based on DSM-IV-TR; International Statistical Classification of Diseases and Related Health Problems, Tenth Revision; and Research Diagnostic Criteria/International Classification of Sleep Disorders, Second Edition criteria: results from the America Insomnia Survey. Biol Psychiatry 2011; 69:592-600.

3. Morin CM, LeBlanc M, Daley M, Gregoire JP, Merette C. Epidemiology of insomnia: prevalence, self-help treatments, consultations, and determinants of help-seeking behaviors. Sleep Med 2006;7:123-130.

4. Ohayon MM, Hong SC. Prevalence of insomnia and associated factors in South Korea. J Psychosom Res 2002;53:593-600.

5. Cho YW, Shin WC, Yun CH, Hong SB, Kim J, Earley CJ. Epidemiology of insomnia in korean adults: prevalence and associated factors. J Clin Neurol 2009;5:20-23.

6. Chung S, Park B, Yi K, Lee J. Pattern of hypnotic drug prescription in South Korea: Health insurance review and assessment service-national patients sample. Sleep Med Res 2013;4:51-55.

7. Youn S, Hann CWC, Park B, Lee S, Kim C, Yi K, et al. The sleeping pill prescription rate for inpatients at a general hospital. Sleep Med Rev 2016; 7:33-38.

8. Ohayon MM, Lader MH. Use of psychotropic medication in the general population of France, Germany, Italy, and the United Kingdom. J Clin Psychiatry 2002;63:817-825.

9. Kyle SD, Morgan K, Espie CA. Insomnia and health-related quality of life. Sleep Med Rev 2010;14:69-82.

10. Ishak WW, Bagot K, Thomas S, Magakian N, Bedwani D, Larson D, et al. Quality of life in patients suffering from insomnia. Innov Clin Neurosci 2012;9:13-26.

11. Ford DE, Kamerow DB. Epidemiologic study of sleep disturbances and psychiatric disorders. An opportunity for prevention? JAMA 1989;262: 1479-1484.

12. Taylor DJ, Mallory LJ, Lichstein KL, Durrence HH, Riedel BW, Bush AJ. Comorbidity of chronic insomnia with medical problems. Sleep 2007; 30:213-218.

13. Engeda J, Mezuk B, Ratliff S, Ning Y. Association between duration and quality of sleep and the risk of pre-diabetes: evidence from NHANES. Diabet Med 2013;30:676-680.

14. Jiao L, Duan Z, Sangi-Haghpeykar H, Hale L, White DL, El-Serag HB. Sleep duration and incidence of colorectal cancer in postmenopausal women. Br J Cancer 2013;108:213-221.
15. Chung S, Youn S, Yi K, Park B, Lee S. Sleeping pill administration time and patient subjective satisfaction. J Clin Sleep Med 2016;12:57-62.

16. Choi JW, Song JS, Lee YJ, Won TB, Jeong DU. Increased mortality in relation to insomnia and obstructive sleep apnea in Korean patients studied with nocturnal polysomnography. J Clin Sleep Med 2017;13:49-56.

17. Belleville G. Mortality hazard associated with anxiolytic and hypnotic drug use in the National Population Health Survey. Can J Psychiatry 2010;55:558-567.

18. Janson C, Lindberg E, Gislason T, Elmasry A, Boman G. Insomnia in men-a 10-year prospective population based study. Sleep 2001;24:425430.

19. Pallesen S, Sivertsen B, Nordhus IH, Bjorvatn B. A 10-year trend of insomnia prevalence in the adult Norwegian population. Sleep Med 2014; 15:173-179.

20. Ford ES, Cunningham TJ, Giles WH, Croft JB. Trends in insomnia and excessive daytime sleepiness among U.S. adults from 2002 to 2012. Sleep Med 2015;16:372-378.

21. Hsu YW, Ho CH, Wang JJ, Hsieh KY, Weng SF, Wu MP. Longitudinal trends of the healthcare-seeking prevalence and incidence of insomnia in Taiwan: an 8-year nationally representative study. Sleep Med 2013; 14:843-849.

22. Lee J, Lee JS, Park SH, Shin SA, Kim K. Cohort Profile: The National Health Insurance Service-National Sample Cohort (NHIS-NSC), South Korea. Int J Epidemiol 2017;46:e15.

23. Keyfitz N. Sampling variance of standardized mortality rates. Hum Biol 1966;38:309-317.

24. Vgontzas AN, Lin HM, Papaliaga M, Calhoun S, Vela-Bueno A, Chrousos GP, et al. Short sleep duration and obesity: the role of emotional stress and sleep disturbances. Int J Obes (Lond) 2008;32:801-809.

25. Bixler E. Sleep and society: an epidemiological perspective. Sleep Med 2009;10(Suppl 1):S3-S6.

26. Ohayon MM, Carskadon MA, Guilleminault C, Vitiello MV. Meta-analysis of quantitative sleep parameters from childhood to old age in healthy individuals: developing normative sleep values across the human lifespan. Sleep 2004;27:1255-1273.

27. Stamatakis KA, Kaplan GA, Roberts RE. Short sleep duration across income, education, and race/ethnic groups: population prevalence and growing disparities during 34 years of follow-up. Ann Epidemiol 2007; 17:948-955.

28. Clark I, Landolt HP. Coffee, caffeine, and sleep: a systematic review of epidemiological studies and randomized controlled trials. Sleep Med Rev 2017;31:70-78.

29. Ohayon MM, Roth T. Place of chronic insomnia in the course of depressive and anxiety disorders. J Psychiatr Res 2003;37:9-15.

30. Johnson EO, Roth T, Breslau N. The association of insomnia with anxiety disorders and depression: exploration of the direction of risk. J Psychiatr Res 2006;40:700-708.

31. Choi WJ, Lee BW, Kim DW, Lee H, Lee S, Oh ST, et al. Current status of insomnia and its comorbidities using National Health Insurance claim data. National Health Insurance Service Ilsan Hospital. Available at: http://www.nhimc.or.kr/cntFileDownLoad.do?DownLoadDir=/ down_data/audit/\&FileName=audit05_etc32.pdf. 2015. Accessed Jan 10, 2017.

32. Kim CS, Ko SH, Kwon HS, Kim NH, Kim JH, Lim S, et al. Prevalence, awareness, and management of obesity in Korea: data from the Korea national health and nutrition examination survey (1998-2011). Diabetes Metab J 2014;38:35-43.

33. Peppard PE, Young T, Barnet JH, Palta M, Hagen EW, Hla KM. Increased prevalence of sleep-disordered breathing in adults. Am J Epidemiol 2013; 177:1006-1014.

34. Chung S, Yoon IY, Lee CH, Kim JW. Effects of age on the clinical features of men with obstructive sleep apnea syndrome. Respiration 2009; 78:23-29.

35. Parthasarathy S, Vasquez MM, Halonen M, Bootzin R, Quan SF, Martinez FD, et al. Persistent insomnia is associated with mortality risk. Am 
J Med 2015;128:268-275. e2.

36. Vgontzas AN, Liao D, Pejovic S, Calhoun S, Karataraki M, Basta M, et al. Insomnia with short sleep duration and mortality: the Penn State cohort. Sleep 2010;33:1159-1164.

37. Lan TY, Zeng YF, Tang GJ, Kao HC, Chiu HJ, Lan TH, et al. The use of hypnotics and mortality-- a population-based retrospective cohort study. PLoS One 2015;10:e0145271.

38. Jaussent I, Ancelin ML, Berr C, Pérès K, Scali J, Besset A, et al. Hypnotics and mortality in an elderly general population: a 12-year prospective study. BMC Med 2013;11:212.

39. Park B, Youn S, Hann C WC, Yi K, Lee S, Lee JS, et al. Prevalence of in- somnia among patients with the ten most common cancers in South Korea: health insurance review and assessment service-national patient sample. Sleep Med Res 2016;7:48-54.

40. Chung S, Bohnen NI, Albin RL, Frey KA, Muller ML, Chervin RD. Insomnia and sleepiness in Parkinson disease: associations with symptoms and comorbidities. J Clin Sleep Med 2013;9:1131-1137.

41. Bryan CJ, Gonzales J, Rudd MD, et al. Depression mediates the relation of insomnia severity with suicide risk in three clinical samples of U.S. military personnel. Depress Anxiety 2015;32:647-655.

42. Zhang B, Wing YK. Sex differences in insomnia: a meta-analysis. Sleep 2006;29:85-93. 